\title{
Tunnel Detection Robot
}

\author{
Nanyu Kuang * \\ North China Electric Power University (Baoding 071000), Hebei, China \\ 531157260@qq.com \\ * the corresponding author
}

Keyword: Wireless communications; Impartial judgment; Tripartite online

\begin{abstract}
At present, the tunnel and tunnel cable maintenance market presents the vigorous development trend in support of national policy. The smart grid construction and downsizing for the needs of social development put forward new requirements for the tunnel cable maintenance industry. We use the power line carrier technology and design the traversal strategy. We rely on wireless communications and wired to carry out data transmission. Based on the above technology, we design and manufacture a cable tunnel inspection robot. Based on the data collected from the robots, we designed an APP for information sharing, enabling tripartite online interaction and impartial judgment of different cables.
\end{abstract}

\section{Research Background}

At present, the national Power Grid Corp has made great efforts to strengthen the forward-looking research in the field of information and communication, deepen the integration of information communication (IT) technology and grid technology, accelerated the construction of a strong smart grid and establish a global energy internet. This series of initiatives coincides with the "Internet + " action plan. By exploring the "Internet" spirit, striving to explore the development mode of integration of IT technology and network technology, it is not difficult to find some new trends of smart grid in future development, and the new demand for IT Technology.

The smart grid is booming today, but none of us notice that underground cables are ignored. Our survey found that in the Transmission and Cable Company's cable tunnel, the working environment is impoverished, the degree of automation is low, and the speed of information transmission is inefficient. Tunnel air quality is poor and it even contains toxic and harmful gases, which are more unsuitable for staff work. At the same time, the timeliness of detection information is weak and the accuracy is impoverished, which greatly affects the timely beginning of safety measures.

Therefore, we propose the "Internet + underground smart grid" company development strategy. First of all, we start from the hardware, independently study and design a cable tunnel inspection robot. This will realize the acquisition of information, replace manual inspections, liberate labor force, and ensure the safety of personnel life. On the basis of the robot, we design an information sharing APP. The information of the robot can be uploaded and displayed on the app. This has realized the timeliness and reciprocity of information, promoted the rapid formation of the national power grid, changed the backward status of underground cable tunnel, and broken the monopoly situation of vicious competition in the wire and cable industry.

\section{Program Introduction}

Online inspection tour based on inspection robots: Patrol robots autonomous inspection as "the first level". That is, the robot can serve as a tool for information discovery, and collect the tunnel information. In this way, we can collect and assemble the cable tunnel information, coordinate the cable status in different coordinates.

The Staff is "the Second Levels". The staff uses APP to upload information from the "first level" to obtain information within its jurisdiction. When an issue occurs, the staff can open the safety system in time, and use the "first level" to obtain the gas information, to analyze whether 
they require help or implement relevant protection. Subsequently, the staff repair the cable appeared obstacles in time, and remediation work. Provided that the staff can not respond promptly, they were likely to apply for other people instead of work. At the same time, APP will detect the corresponding time, and if it is too long, it will automatically notify the staff that is closest to the problem cable.

Resident users are "the third levels". Residents are supervision side, when the "first level" and the "second level" alarm can not make a positive response, the "third levels" can use APP to protect their own interests and request other staff to make maintenance or contact with a higher level of cable tunnel management;

Healthy Competition Scheme of Wire and Cable Industry Based on APP Data Analysis. APP uploads data into categories, wires and cables produced by different companies, and they have different signs. When there is trouble, APP will do online records and share them with other three parties. Power Grid Corp, cable companies and user groups all get the same information.

According to the above conditions, the products in the wire and cable industry can be transparent and share the information avoid of the emergence and development of regional monopoly.

\section{Product introduction}

Product Structure. The robot includes a control system, a motion actuator, a gas detection device, a cable detection device, a navigation, a positioning device, a communication device and a power management system. The block diagram of the moving body is shown in Fig. 1. The inspection system includes the robot (moving body), the communication network and the remote terminal.

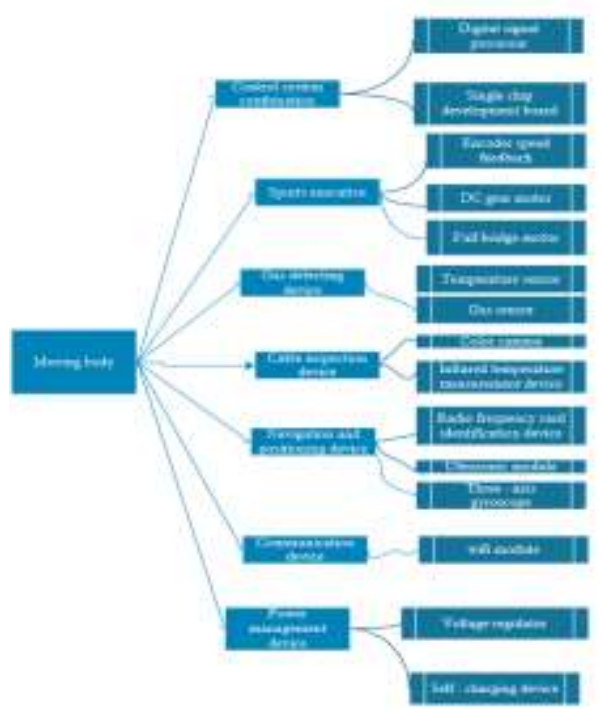

Figure 1. Partition diagram of moving body combination

\section{Motion Control Part of the Working Principle}

Motor and Drive Module. We analyze the working conditions of the cable tunnel robots and conclude that the robot is required to complete the low-speed forward movement, turning it in situ and adapting to the slope ground. In order to meet the above requirements, the design uses four-wheel drive structure, with a full bridge motor drive module control DC gear motor, to achieve low-speed motor forward and reverse. The schematic diagram of the full bridge drive is shown in Fig. 2. The DC reduction motor comes with a reducer, which has low speed and high torque. It is suitable for the cable tunnel robot. The parameters of the DC speed reduction unit are: $\mathrm{DC}=24 \mathrm{~V}$, $\mathrm{n}=194 \mathrm{r} / \mathrm{min}, \mathrm{nN}=155 \mathrm{r} / \mathrm{min}, \mathrm{In}=0.6 \mathrm{~A}, \mathrm{Tn}=12 \mathrm{~kg} / \mathrm{cm}, \mathrm{Id}=2 \mathrm{~A}, \mathrm{Td}=26 \mathrm{~kg} \bullet \mathrm{cm}$. 


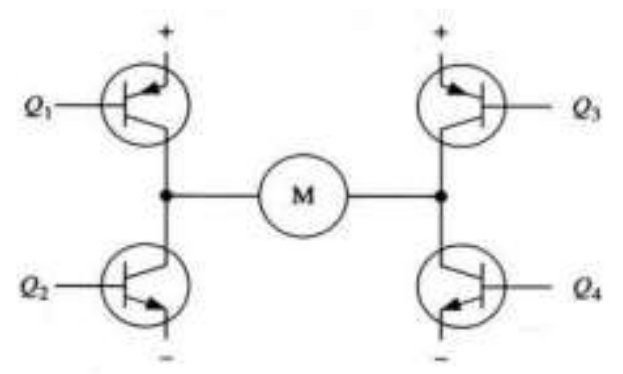

Figure 2. Schematic diagram of full bridge circuit

Transistor allows less current, so the drive chip needs to use high-power MOS. Moreover, we need to match the isolation chip to make the motor driver board. As shown in Fig.2.3, when the Q1, Q4, Q2 and Q3 are disconnected, the motor rotates, which specifies that the turn is positive. When the Q1 and Q4 are disconnected, the Q2 and Q are turned on and the motor turns backward. However, if the Q1 and Q2 are simultaneously connected, the circuit will short-circuit and burn down the power source, which is not allowed. If the hardware does not add protection, it can only rely on programmers to increase their attention and avoid errors in the program. To be on the safe side, we have set the driver protection circuit for the drive board to avoid the above situation. CON1 and CON2 are used to indicate two control signals. In order to prevent short circuits, we need to set up logical circuits to eliminate the Q1 and Q2, all of which are 1, and retain the other three combinations $(1,0),(0,1)$ and $(0,0)$. We use NAND and NOR gates, the design of logic circuit diagram as shown in figure 3 . The actual circuit design, using 74HC00 chip and 74HC04 chip NAND gate, completes the circuit diagram. The logical table is shown in table 2.1.

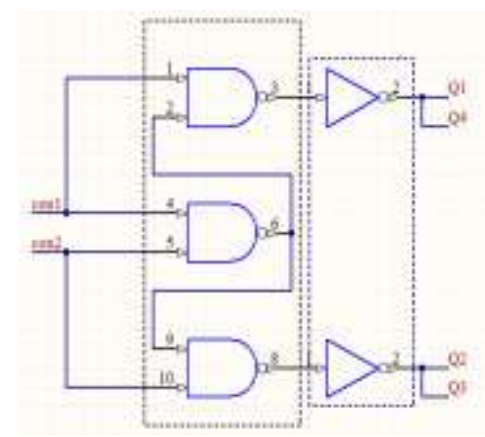

Figure 3. Drive protection logic circuit

Table 1 Drive and protect circuit logic table

\begin{tabular}{|c|c|c|c|c|}
\hline Con Q & Q1 & Q2 & Q3 & Q4 \\
\hline$(\mathbf{0 , 0}$ & 0 & 0 & 0 & 0 \\
\hline$(\mathbf{0 , 1})$ & 0 & 1 & 1 & 0 \\
\hline$(\mathbf{1 , 0})$ & 1 & 0 & 0 & 1 \\
\hline$(\mathbf{1 , 1})$ & 0 & 0 & 0 & 0 \\
\hline
\end{tabular}

Principle and Algorithm of Speed Regulation. SCM control the speed of the 4 motors through the output of PWM square wave signal. The duty cycle of PWM square wave is linearly related to the speed, which is the principle of motor speed regulation by using PWM. Considering that Q1 and Q4 have the same on-off state, that is, logical synchronization. Therefore, we only need a control line to control Q1 and Q4 at the same time. In other words, we only need 2 PWM to realize the positive and negative speed control of an electric motor. The same side motor output the same speed, so we only need 4 PWM to complete the control of the 4 motors. When the robot goes forward, we need equal left and right wheel speeds to maintain the direction of travel. Considering the large error of the open-loop speed regulation, we use the closed-loop speed feedback system. At the same time, we control the motor speed with the PID adjustment method. Concretely, we use 
encoder to speed the motor, and calculate the difference between the output speed of the encoder and the given speed speed-n:

cha=speed_n.speed

Then enlarge the difference (the magnification needs to be adjusted) and integrate:

speed_pi=kp*cha+speed_pi

We calculate the difference between the two adjacent differences and enlarge

(the magnification needs actual adjustment):

speed_d=ki*(cha.cha_last)

Calculates the current output pwm_value:

pwm_value $=\mathrm{ks} *($ speed_pi+speed_d $)$

Finally, we update the duty cycle of the PWM waveform.

\section{Design of Positioning and Navigation System}

Autonomous Direction Correction. Cable tunnel robot without rail style: it uses ultrasonic sensor to measure the distance of robot and the tunnel side wall, and the robot according to the return distance value determines the direction of deviation and position deviation. Ultra sonic sensor works as follows: the control port sends more than one 10us: high level, you can wait for the high level output at the receiving port. Once the sensor has an output, the robot can turn on the timer. When this port is low, you can read the value of the timer, which is the range of time to calculate the distance.

We use a microcontroller to retrieve the distance from an ultrasonic sensor: first we set up a timer, its counting cycle is $1 \mathrm{~s}$, and each cycle counts 106 numbers, that is, the timer count every lus. In addition, we set an I/O pin that operates in the input mode and which is equipped with the rising edge to trigger the interrupt callback function raising (). When the receive port is pulled high, the system enters the interrupt function raising () and reads the timer's current count value t0, configuring the falling edge to trigger an interrupt callback function falling (). When the receive port is pulled low, the system enters the interrupt function falling (), reads the current count value t1 of the timer, and configures the rising edge to trigger an interrupt callback function raising ().Sound speed is $34000 \mathrm{~cm} / \mathrm{s}$, the distance (unit is $\mathrm{cm}$ ) is calculated as:

$(\mathrm{t} 1+1000000 . \mathrm{t} 0) / 1000000 * 34000 / 2$

Considering the spatial characteristics of the tunnel, we use multiple ultrasonic sensors to detect the robot from the wall, considering the information from multiple sensors, so that we can judge between the robot and the wall of tunnel and the distance of the moving body and the direction of tilt angle. The layout of ultrasonic sensors is: An ultrasonic sensor is arranged in the direction of the front of the shell, and two equal spacing ultrasonic sensors are arranged on the left side and in the parallel direction. An ultrasonic sensor in the forward direction is used to detect whether there is a wall in front. The sensors are used to calculate the shortest distance between the robot and the wall so that we can calculate the wei_error between the robot and the centerline of the road:

$$
\text { dir_error=arcsin((R2.R1)/Long) }
$$

The microcontroller uses the position offset and angle offset of the robot to correct it. The sketch map of robot offset calculation is shown in Fig. 2.7. 


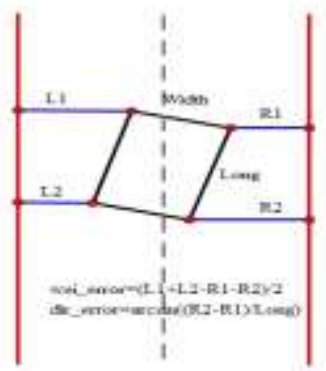

Figure 4. Offset diagram

\section{Data Transfer Part of the Working Principle}

Power Carrier. Power line carrier communication PLC is the abbreviation of Power line Communication. Power line carrier is the unique communication mode of power system. Power line carrier communication is a technology that uses existing power lines to transmit analog signals at high speed. The biggest feature is that there is no need to reset the network, on condition that there is wire, you can transfer data.

The power carrier communication equipment we use is HL. PLC embedded power line carrier module. Fig. 2.19 is a physical picture of the module. The module uses fuzzy algorithm. Its anti-interference ability is strong, communication distance is far, it adapts to China's domestic power line application environment. Baud rate of serial port of communication module can be set by user (default 9600), even parity check and no check option. They can be directly connected to the RXD and TXD of the microcontroller to complete the transparent forwarding of data from the serial port to the carrier. HL.PLC carrier communication module uses FSK communication, and software uses super fuzzy algorithm, even if the transmission signal is disturbed or lost $40 \%$, the original carrier signal can also be accurately restored. In addition, the communication is stable and anti-interference capability is strong. The carrier center frequency is $72 \mathrm{~K}$; the carrier line effective data rate can reach $6.7 \mathrm{Kbit} / \mathrm{s}$ in normal transmission mode and $1 \mathrm{Kbps}(1024 \mathrm{byte} / \mathrm{s})$ in the environment of $50 \mathrm{~Hz}$ communication point. The module is fully transparent and has no character length limit. They can directly replace half duplex communications such as 485 and 232 . The carrier communication port can directly resist the impact of static electricity, group pulse and surge.

The power carrier equipment and the $\mathrm{WiFi}$ module are connected by serial port. It can realize data exchange with WiFi module carried by robot. It is important to note that the power supply between the communicated equipment at the intersection and the power supply of the robot is independent of each other. The power supply of the power line carrier equipment is supplied by low-voltage power lines.

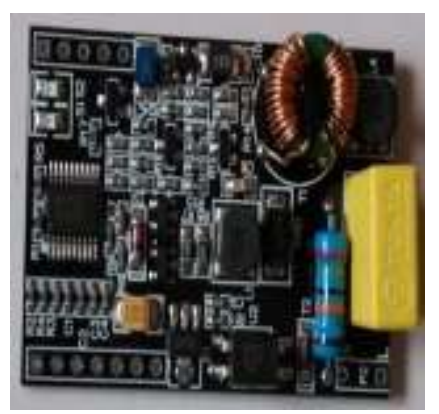

Figure 5. Power Carrier Communication Module

The WiFi module rated voltage is $3.3 \mathrm{~V}$, we need to set the buck module to reduce the DC9V to DC3.3V. WiFi module power is low, the current requirements are small, and so the use of more common AMS1117 chip can meet the requirements here. AMS1117.3.3 voltage regulator chip: fixed voltage is $3.3 \mathrm{~V}$, the maximum output current is $1 \mathrm{~A}$, line regulation rate: $0.2 \%$ (maximum), 
load regulation rate: $0.4 \%$ (maximum), package type: SOT.223. Figure 6 is the physical and electrical schematics of the AMS1117 chip. [2]
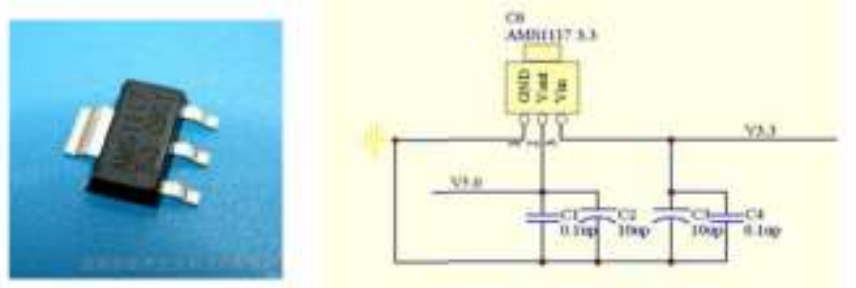

Figure 6. AMS1117 physical map and electrical schematic

\section{References}

[1] Sui Feng Jing. The tunnel lining quality detection technology [J]. engineering, 1998, (02): 67-69. [2017-10-07].

[2] Jiang Yun, Fu Zhuang. Design of a small cable tunnel inspection robot [J]. East China power, 2009,37 (01): 95-97. [2017-10-07].

[3] Xie Xie. Research on control system of integrated inspection robot for cable tunnel [D]. Shanghai Jiao Tong University, 2008.

[4] Fu Ruhui. Small tunnel detection crawler robot [J]. Equipment Manufacturing Technology, 2010, (03): 21-22. [2017-10-07].

[5] Du Yigang. Cable tunnel inspection robot development [D]. Inner Mongolia University of Technology, 2014. 